EUROPEAN ORGANIZATION FOR NUCLEAR RESEARCH

TIS-RP $/ 176 / C F$

7 October 1986

\title{
SIMULTANEOUS DETERMINATION OF DOSE AND ELAPSED TIME IN ACCIOENT DOSIMETRY USING THERMOLUMINESCENT MATERIALS
}

\author{
C. Furetta*, J.W.N. Tuyn and F. Louis \\ CERN, Geneva, Switzerland \\ $\mathrm{J}$. Azorin \\ Instituto Nacional de Investigaciones Nucleares \\ Mexico City, D.F. Mexico \\ C.M.H. Driscoll \\ National Radiological Protection Board \\ Chilton, Didcot, UK
}

\section{ABSTRACT}

Information about the time which has elapsed since an external radiation exposure is useful in determining the time of occurrence of an abnormal exposure in personal and accident dosimetry. This information can be obtained from certain irradiated thermoluminescent dosemeters. A computational method based on decay rates of thermoluminescence glow peaks has been used to estimate a range of suitable values of trap lifetimes of low temperature glow peaks. This provides a basis for the selection of an appropriate thermoluminescent material to assess not only the absorbed dose from a radiation exposure but also the elapsed time. The results of experimental studies have been used to obtain additional information on decay rates as a function of energy, temperature, and absorbed dose.

To be presented at

8th International Conference on Solid State Dosimetry

Oxford, 26-29 August 1986

\footnotetext{
On leave from Dept. of Medical Physics, Rome University, "La Sapienza", Rome.
} 


\section{INTRODUCTION}

The glow-curve of a thermoluminescent material may consist of several glow peaks, each having its maximum signal at a different temperature. Those peaks which occur in the temperature region above $453^{\circ} \mathrm{K}$ are generally thermally stable at normal post-irradiation storage temperatures, and the integrated area or the height of such a peak is used to asses the absorbed dose from a radiation exposure. However, in many materials there are less thermally stable peaks whose temperatures lie below $453^{\circ} \mathrm{K}$. By using the decay rates of suitable lower temperature peaks, the elapsed time since an abnormal or accidental exposure may be determined.

In the present study a computer-based analysis has been used to extend the mathematical model reported previously [1] to estimate a range of suitable values of the lifetimes of low-temperature glow peaks appropriate to several commonly used thermoluminescent materials. The theoretical model is then compared with experimental data on various preparations of calcium sulphate $\left(\mathrm{CaSO}_{4}\right)$ and calcium fluoride $\left(\mathrm{CaF}_{2}\right)$.

In setting up the theoretical model, an idealized thermoluminescent material is considered which has two well-separated peaks at temperatures $T_{1}=373^{\circ} \mathrm{K}$ and $T_{2}=473^{\circ} \mathrm{K}$.

The decay rate of the less stable lower temperature peak can be related to the mean electron lifetime $\tau$ in a trap corresponding to the peak as follows:

$$
\begin{array}{ll}
\tau=s^{-1} \exp (E / k T) & \text { 1st order kinetics [2] } \\
\tau^{*}=\left(s^{\prime} n_{0}\right)^{-1} \exp (E / k T) & \text { 2nd order kinetics [3] }
\end{array}
$$

where $s$ is the frequency factor $\left(s^{-1}\right)$;

$\mathrm{s}^{\prime}$ a pre-exponential factor $\left(\mathrm{m}^{3} \mathrm{~s}^{-1}\right)$;

$n_{0}$ the initial concentration of trapped electrons $\left(m^{-3}\right)$;

$E$ the activation energy for thermal release of charge (eV);

$k$ Boltzmann's constant and

$T$ the temperature $(K)$. 
The light or integrated area under a glow peak t at a time $t$ after irradiation is expressed as:

$$
\begin{array}{ll}
+=t_{0} \exp (-t / \tau) & \text { 1st order kinetics } \\
+=t_{0}\left(1+t / \tau^{\star}\right)^{-1} & \text { 2nd order kinetics }
\end{array}
$$

These equations describe the glow peak resulting from a single instantaneous exposure occurring at time $t=t_{i}$, where $\downarrow_{0}$ is the glow peak area at time $t=0$.

In the present model, the instantaneous exposure is superimposed on a continuous and constant background signal B (i.e. an environmental background exposure) such that

$$
\begin{array}{lll} 
& =\alpha B \tau[1-\exp (-t / \tau)] & \text { 1st order kinetics } \\
\phi=\left[\left(\alpha B \tau^{*}\right)^{1 / 2} \tanh \left[\left(\alpha B / \tau^{*}\right)^{1 / 2} t\right]\right. & \text { 2nd order kinetics }
\end{array}
$$

where $B$ is the rate of increase of the background signal;

$\alpha$ is the thermoluminescence sensitivity for a particular material (i.e. the thermoluminescence signal per unit absorbed dose and

$$
\tanh \left[\left(\alpha B / \tau^{*}\right)^{1 / 2} t\right]=\frac{\left.1-\exp \left[-2\left(\alpha B / \tau^{*}\right)^{1 / 2} t\right)\right]}{\left.1+\exp \left[-2\left(\alpha B / \tau^{*}\right)^{1 / 2} t\right)\right]}
$$

Combining Eqs. (1) and (3), and (2) and (4), and expressing the results as a ratio $R$ of $\phi_{2}$ for peak 2 (stable) and over $\phi_{1}$ for peak 1 (unstable), the following expressions are obtained:

$$
\begin{array}{r}
R=\frac{\phi_{2}}{\phi_{1}}=\frac{\left(\phi_{0}\right)_{2} \exp \left[-\left(t-t_{i}\right) / \tau_{2}\right]+\alpha_{2} B \tau_{2}\left[1-\exp \left(-t / \tau_{2}\right)\right]}{\left(\phi_{0}\right)_{1} \exp \left[-\left(t-t_{i}\right) / \tau_{1}\right]+\alpha_{1} B \tau_{1}\left[1-\exp \left(-t / \tau_{1}\right)\right]} \\
\text { 1st order kinetics } \\
R=\frac{\phi_{2}}{t_{1}}=\frac{\left.\left(\phi_{0}\right)_{2}\left[1+\left(t-t_{i}\right) / \tau_{2}^{*}\right]^{-1}+\left(\alpha_{2} B \tau_{2}^{*}\right)^{1 / 2} \tanh \left[\alpha B / \tau_{2}^{*}\right)^{1} t\right]}{\left.\left(\phi_{0}\right)_{1}\left[1+\left(t-t_{i}\right) / \tau_{1}^{*}\right]^{-1}+\left(\alpha_{1} B \tau_{2}^{*}\right)^{1 / 2} \tanh \left[\alpha B / \tau_{1}^{*}\right)^{1} t\right]} \\
\text { 2nd order kinetics }
\end{array}
$$

$R$ from equations [5] and [6] has been plotted against elapsed time $\left(t-t_{i}\right)$ in Fig. 1 for some values of the mean trap lifetimes using the following physical parameters: $\left(\phi_{0}\right)_{1}=90,\left(\phi_{0}\right)_{2}=100, \alpha_{1}=90$, $\alpha_{2}=100$ ( $\mathrm{TL}$ counts $\left.\mathrm{mGy}^{-1}\right), B=3 \times 10^{-3}$ (mGy.day $\left.{ }^{-1}\right), t=30$ days and $\tau_{2}=\tau_{2}^{*}=400$ days. An abnormal exposure can occur from day 0 up to 
day 30, the thermoluminescent dosemeters being read on day 30 . If the time elapsed is zero (i.e. $t=30$ days), there is no fading of the first low temperature peak and the peak ratio is small. If the abnornal exposure occurs at the beginning of the monitoring period, the peak ratio is high. For practical application, to ascertain precisely the elapsed time since an abnormal exposure, a steep gradient is required in the plot of $R$ against $\left(t-t_{i}\right)$. For first order kinetics this is achieved with trap lifetimes in the range 4 to 12 days [1]. Mean trap lifetimes for low temperature peaks in some thermoluminescent materials have been published [4]. LiF:Mg, Ti and $\mathrm{CaSO}_{4}: \mathrm{Mn}$ are reported to have mean trap lifetimes of -1.5 days for the lower temperature peaks $\left(\sim 383^{\circ} \mathrm{K}\right)$ which are lower than the values predicted as suitable by the model. CaSO $_{4}:$ Sm has a mean trap lifetime of $1.5-3$ days, which approaches the suitable computed values, while CaF :Dy with a mean trap lifetime of 10 days appears the most suitable from reported data.

\section{EXPERIMENTAL METHOD AND RESULTS}

Experiments to test the validity of the theoretical model have been made using the thermoluminescent materials listed in Table 1 with appropriate experimental parameters. Following annealing, all samples were irradiated with a test dose of $50 \mathrm{mGy}\left({ }^{137} \mathrm{Cs}\right)$. After readout in a manual Harshaw reader (model 2000) which was coupled to a glow-curve analyser (model 2080), only samples with a response within $\pm 5 \%\left(\begin{array}{ll}1 & 0\end{array}\right)$ from the mean of a batch were selected and then individually calibrated. A light-source correction was used for changes in photomultiplier tube sensitivity. A linear heating rate of $10 \mathrm{K.s}{ }^{-1}$ from room temperature to $573^{\circ} \mathrm{K}$ was used in a readout time of $40 \mathrm{~s}$. Data on the total light as the integral of the glow-curve area, the partial glow-peak areas and the glow-peak heights were obtained. Each experimental data point represents the mean of at least five dosemeters. Dosemeters were stored in the dark at an average ambient temperature of $293^{\circ} \mathrm{K}$. The environmental dose rate was $2-3 \mu \mathrm{Gy}$.day ${ }^{-1}$. After different periods of storage during the monitoring period groups of dosemeters were irradiated to simulate an accidental exposure and were then stored again. At the end of the monitoring period all dosemeters were read along with zero-dosed background controls. 
An example of the experimental results for $\mathrm{CaSO}_{4} / \mathrm{LiF}: \mathrm{Dy}$ and CaSO $_{4}$ :Dy (TLD 900) given an abnormal dose of $1 \mathrm{~Gy}$ is shown in Fig. 2, where the plots of the peak height ratio and the peak area ratio are given as functions of the elapsed time since irradiation. The trend of the experimental plots are similar to the theoretical curves of Fig. 1 and seems to follow a 2nd order kinetics. In fact, the kinetics of $\mathrm{CaSO}_{4}$ is reported to follow an intermediate order $[5,6]$. The best fit to the experimental data is represented by a 4th degree polynomial using the Tchebychev approximation. For the data shown in Fig. 2 the elapsed time can be related to the peak area ratio (i.e. the $t_{\text {}}$ equations) or the peak height ratio (i.e. the $t_{H}$ equations) as follows: $\mathrm{CaSO}_{4} / \mathrm{LiF}: \mathrm{DY}$

$$
\begin{aligned}
& t_{\phi}=-194+381 R_{\phi}-262 R_{\phi}^{2}+75.4 R_{\phi}^{3}-7.4 R_{\phi}^{4} \\
& t_{H}=12470-44310 R_{H}+58780 R_{H}^{2}-34540 R_{H}^{3}+7600 R_{H}^{4}
\end{aligned}
$$

$\mathrm{CaSO}_{4}:$ Dy (TLD 900)

$$
\begin{aligned}
& t_{\phi}=73.9-86.3 R_{\phi}+34.8 R_{\phi}^{2}-5.8 R_{\phi}^{3}+0.35 R_{\phi}^{4} \\
& t_{H}=8890-14780 R_{H}+9140 R_{H}^{2}-2496 R_{H}^{3}+254.5 R_{H}^{4}
\end{aligned}
$$

Similar results for these materials to those shown in Fig. 2 have been obtained for abnormal exposures to ${ }^{137} \mathrm{Cs}(200 \mathrm{mGy})$ and PuBe neutrons (46 mGy). Exposure to ${ }^{241}$ Am lower energy photons (60 kev) (78 $\mathrm{mGy}$ ) showed that the gradients of the plots of $R$ against elapsed time are less steep compared to the higher energy photon and neutron irradiations.

The effect of storage temperature on the peak ratios has been studied by storing CaSO $/$ LiF:Dy at a high temperature of $313^{\circ} \mathrm{K}$ during the monitoring period. As a result of thermal fading the elapsed time determination is limited to up to 15 days after irradiation.

Similar experiments have been performed on $\mathrm{CaSO}_{4} / \mathrm{KBr}: \mathrm{DY}$ and $C_{2}$ :DY (TLD 200); :Tm (TLD 300). The usefulness of the former material to elapsed time studies is limited to a period less than 15 days from readout. The plots for $\mathrm{CaF}_{2}$ appear too flat to be useful at room temperature but show more marked changes particularly in $R_{\phi}$ at $313^{\circ} \mathrm{K}$. 


\section{SUMMARY}

Experimental data on $\mathrm{CaSO}_{4} / \mathrm{LiF}$ :Dy and $\mathrm{CaSO}_{4}$ :Dy (TLD 900) agree favourably with theoretical predictions, particularly in the variation of peak area ratio with elapsed time since an abnormal exposure superposed on a constant and continuous background exposure. The peak area ratio is found to be more useful than the peak height ratio because the elapsed time can be estimated with a smaller error and the better precision is obtained for elapsed times of less than 15 days. A polynomial fit to the experimental data gives the difference between the experimental and calculated elapsed times using $R$ of about 1 day. The gradients of $R$ against elapsed time are less steep for low energy photon irradiations than for high energy photon and neutron irradiations. For TLD 900 the error in time determination for an irradition with ${ }^{241}$ Am from 5 to 50 days is \pm 4 to 5 days. Further studies on the effect of high storage temperatures and at different absorbed doses will be reported later. 


\section{REFERENCES}

1. C. Furetta, C.M.H. Driscoll, R. Pani, R. Pellegrini, A computational method to assess the time elapsed since exposure using TL dosemeters. To be published in Radiation Effects (1986).

2. J.T. Randall and M.H.F. Wilkins, Phosphorescence and electron traps, Proc. R. Soc. 184A, 365 (1945).

3. S.F.J. Garlick and A.F. Gibson, The electron trap mechanism of luminescence in sulphide and silicate phosphors, Proc. Phys. Soc. $\underline{60}, 574$ (1948)

4. M.W. Charles, An extended role for thermoluminmescent phosphors in personnel, environmental and accident dosimetry using sensitisation, re-estimation and fast fading, Nucl. Instr. Methods 206, 239 (1983).

5. C. Furetta and P. Gennai, Rinetics and thermoluminescent parameters of $\mathrm{CaSO}_{4}:$ Dy ribbon, TLD-900, at low dose levels, Radiation Effects $\underline{55}, 23$ (1981).

6. J. Azorin and A. Gutierrez, Determination of TL parameters in $\mathrm{CaSO}_{4}$ :DY, Nucl. Sc. J. 22, 305 (1985). 


\section{FIGURE CAPTIONS}

Fig. 1 Peak area ratio against elapsed time predicted by the theoretical model as a function of trap lifetime.

Fig. 2 Experimental data on peak height and peak area ratios against elapsed time for $\mathrm{CaSO}_{4} / \mathrm{LIF}: \mathrm{DY}$ and $\mathrm{CaSO}_{4}$ :Dy (TLD 900) dosed to $1 \mathrm{~Gy}\left({ }^{137} \mathrm{Cs}\right)$. 
TABLE 1

Information on thermoluminescent materials and experimental parameters used in this study

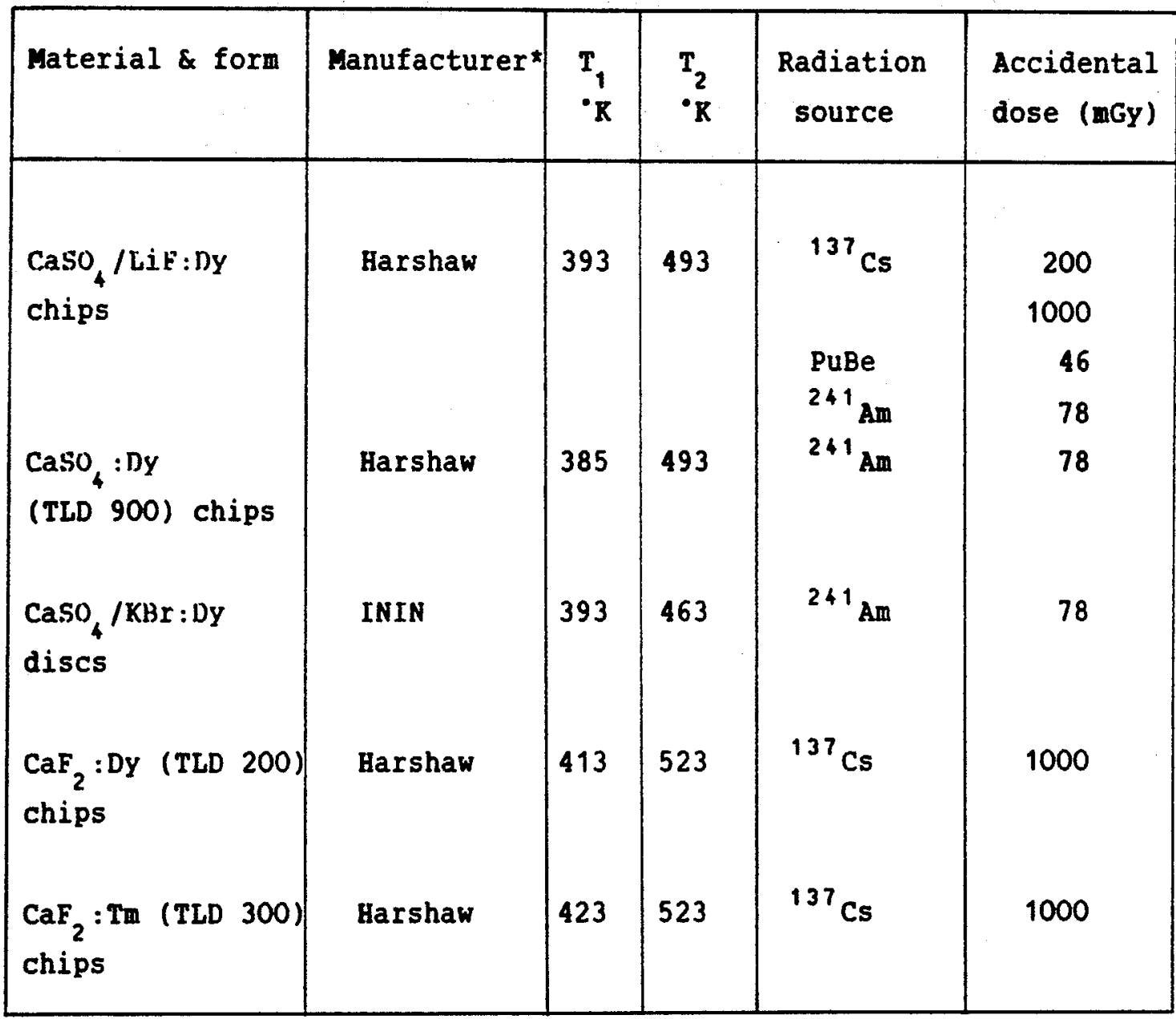

* Harshaw refers to the Harshaw Chemical Co., Cleveland, Ohio, USA, ININ refers to the Instituto Nacional de Investigationes Nucleares, Mexico City, Mexico. 


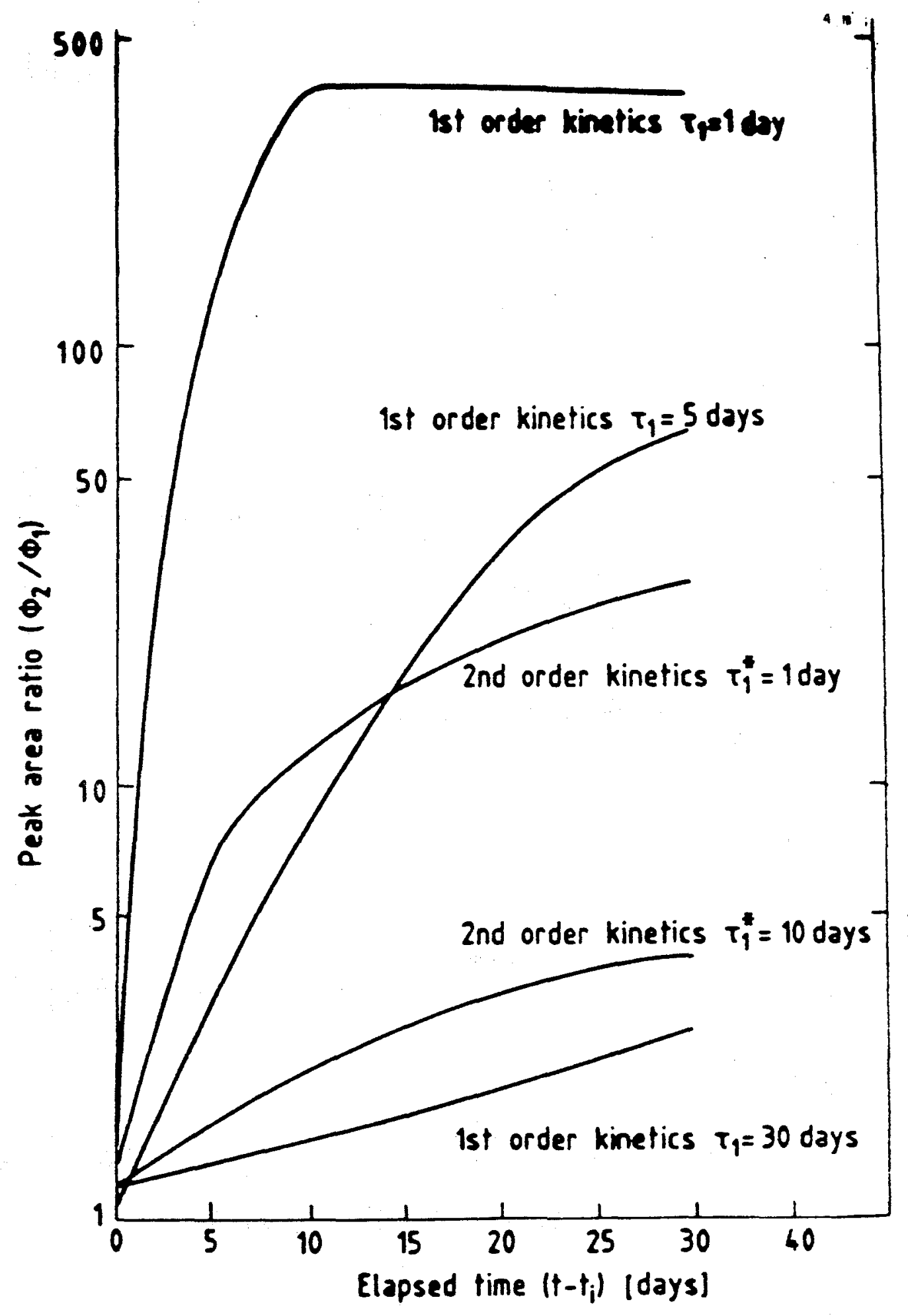

Fig. 1 


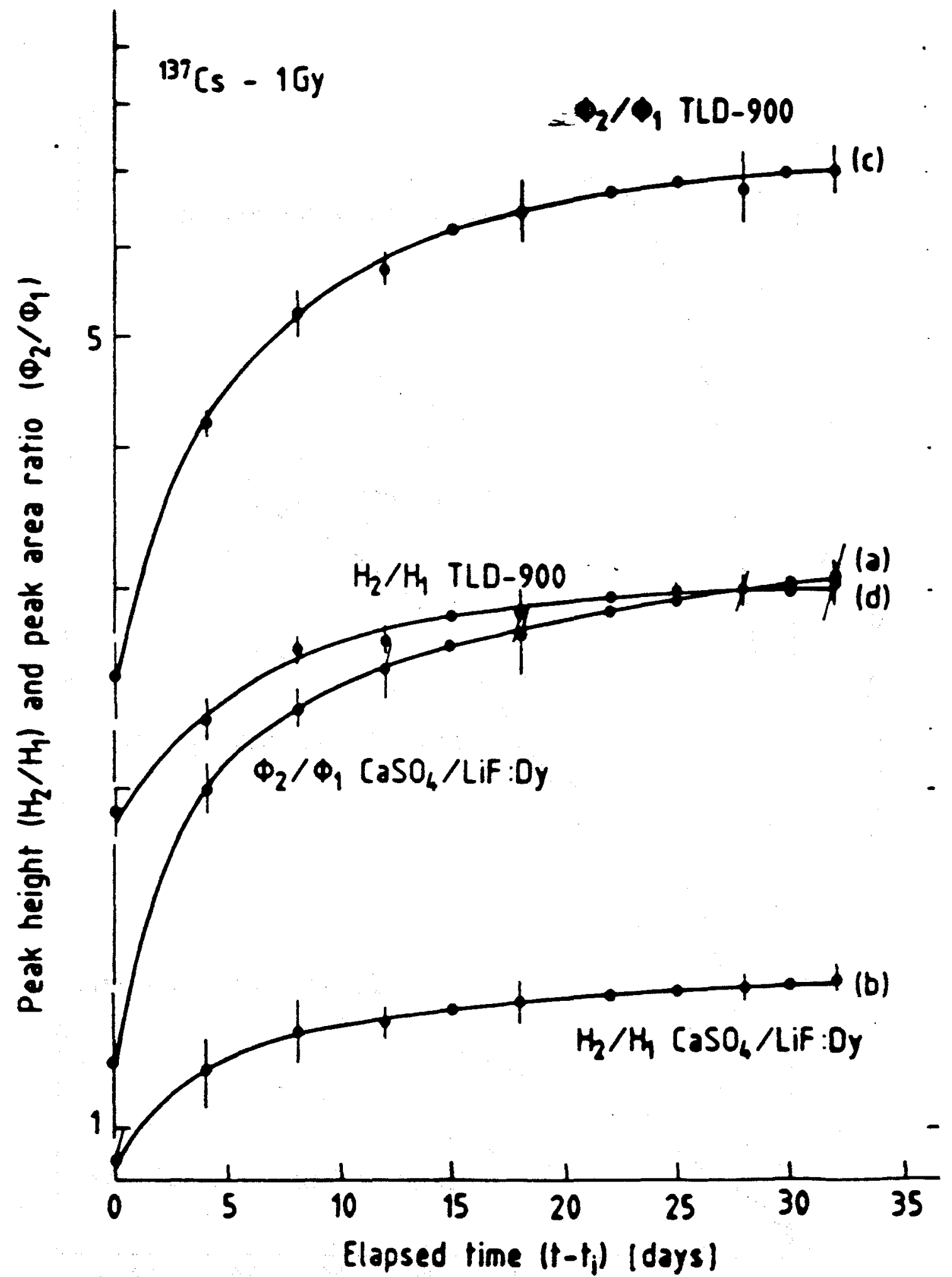

Fig. 2 